\title{
The Effect of Brain-Derived Neurotrophic Factor Val66met Polymorphism on Acquisition and Motor Memory Consolidation
}

\section{Abolfazl Shayan Nooshabadi ${ }^{1}$, Alireza Saberi Kakhki ${ }^{*}$, Mehdi Sohrabi ${ }^{1}$, Mohamad Ali Dowlati ${ }^{2}$ \\ ${ }^{1}$ Department of Motor Behavior, Faculty of Sport Sciences, Ferdowsi University of Mashhad, Mashhad, Iran}

${ }^{2}$ Research Center for Biochemistry and Nutrition in Metabolic Diseases, Kashan University of Medical Sciences and Health Services, Kashan, Iran

\section{A BSTRACT}

Introduction: Brain-derived neurotrophic factors (BDNF) play a role on induction and maintenance of memory and learning. The BDNF gene Val66Met polymorphism impairs expression of this protein. Due to difference between the neural principles operating during motor memory consolidation and the neural principles operating during practice (acquisition) and the effect of BDNF on both of these processes, the aim of the study was to study the effect of val66met polymorphism on acquisition and motor memory consolidation in the process of learning of throwing motor skill. Materials and Methods: We randomly selected one hundred university students of Kashan , Iran (mean age: $21.6 \pm 2.2$ years). After blood sampling, extraction of genomic DNA, implementation of polymerase chain reaction, analyzing of PCR by 1.5 percent Electrophoresis Gel, and in the end sequencing by ABI PRISM 7000 Sequencing Analyzer, 46 participants were identified without val66met polymorphism, and 54 participants identified affected by the polymorphism (met-carriers). 10 participants of each genetic group (20 people) after pre-test practiced backhand baseball pitch for six sessions. After 48 and 96 hours they participated in two consecutive retention tests. Results: Our data showed no difference between met-carriers and people without the polymorphism in acquisition and the first retention test. However, met-carriers were significantly weaker than people without the polymorphism in the second retention test. Conclusion: These findings suggest that the BDNF val66met polymorphism has higher impact on motor learning in the consolidation phase than acquisition phase.

\section{Key words:}

1. Brain-Derived Neurotrophic Factor

2. Polymorphism, Single

Nucleotide

3. Learning

*Corresponding Author: Alireza Saberi Kakhki

E-mail:askakhki@um.ac.ir

doi: 10.18869/acadpub.shefa.4.4.10 


\title{
تأثير يلىمورفيسم val66met فاكتور نوروتروفيك مشتق از مغز بر اكتساب و تحكيم سازى حافظئ

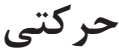

\author{
ابوالفضل شايان نوش آبادى'، عليرضا صابرى كاخكى"'، مهدى سهر ابى'، محمدعلى دولتى' \\ اگروه رفتار حركتى، دانشكده علوم ورزشى، دانشعاه فردوسى مشهد، مشهد، ايران \\ 'مركز تحقيقات بيوشيمى و تغذيه در بيمارىهاى متابوليك، دانشگاه علوم يزشكى و خدمات بهداشتى درمانى كاشان، كاشان، ايران
}

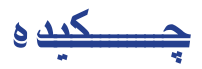

مقدمه: فاكتورهاى نوروتروفيك مشتق از مغز در القاء و نكهدارى حافظه و يادكيرى نقش ايفاء مى كند.

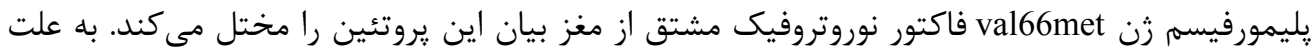

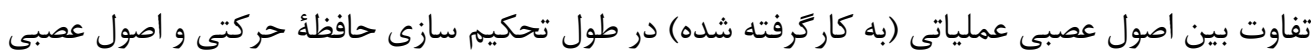

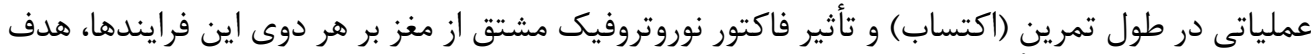

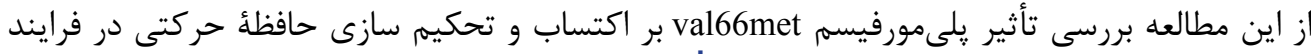

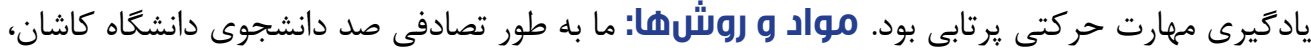

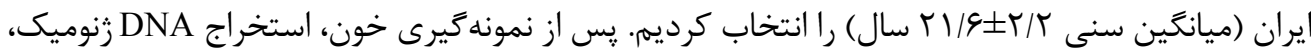

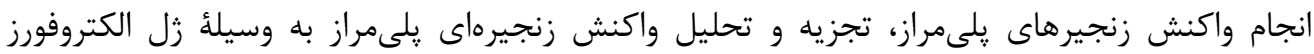

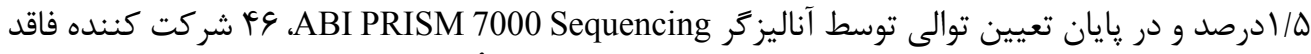

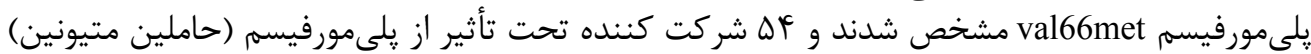

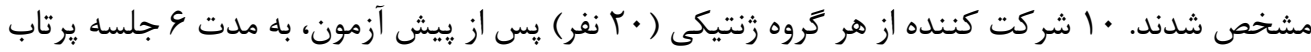

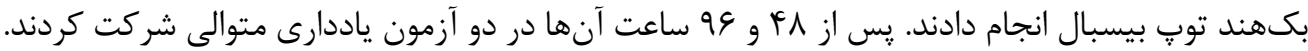

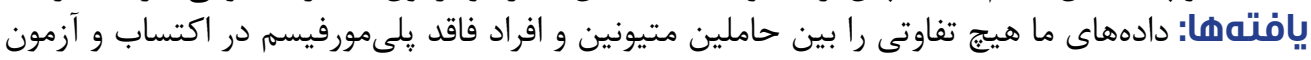

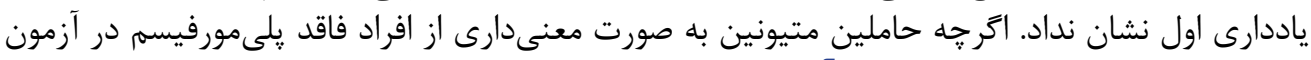

كليد وازمها:

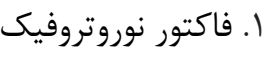

مشتق از مغز

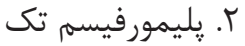

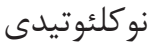

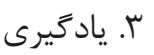

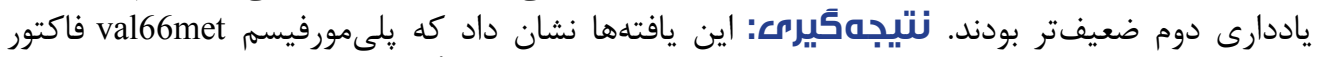

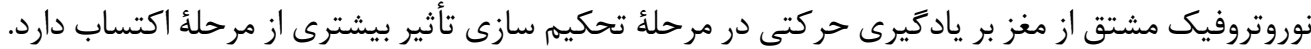




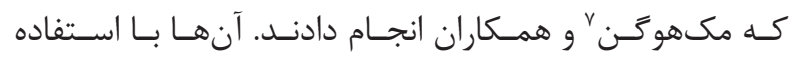

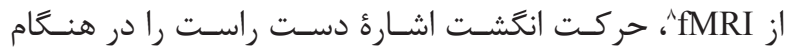

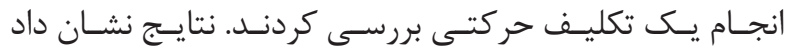

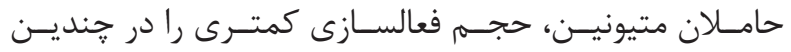

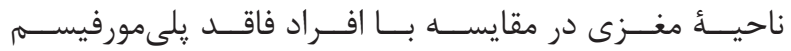

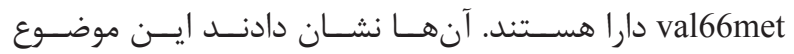

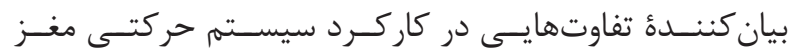

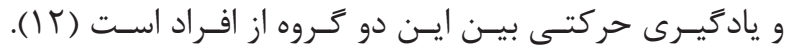

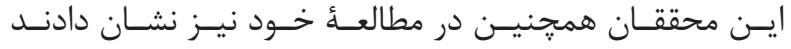

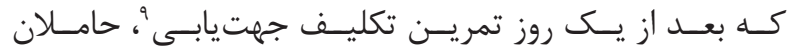

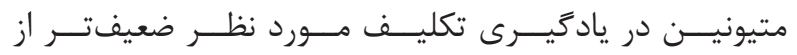

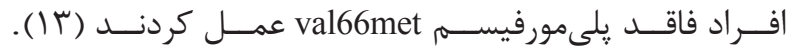

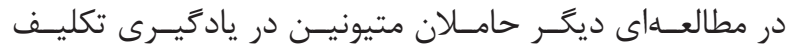

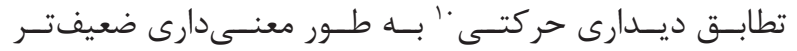

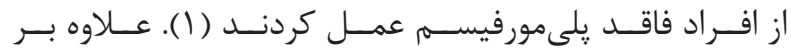

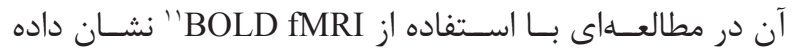

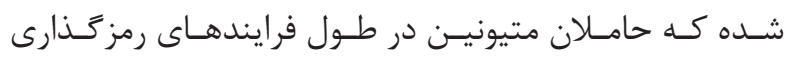

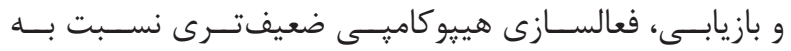

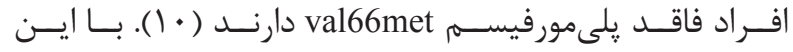

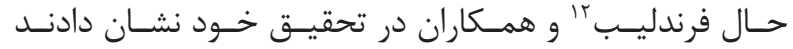

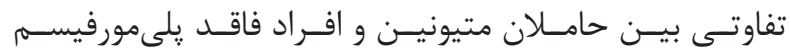

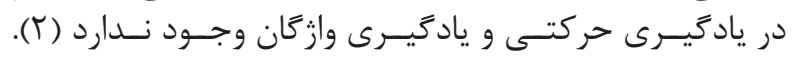

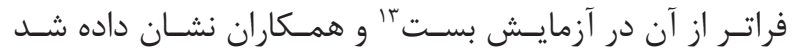

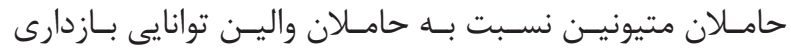

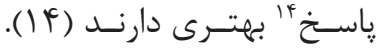

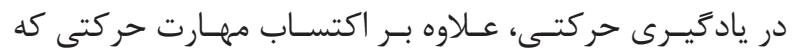

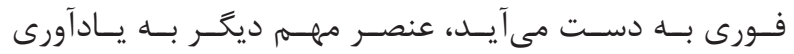

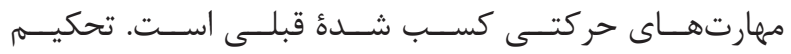

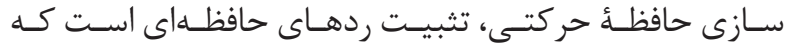

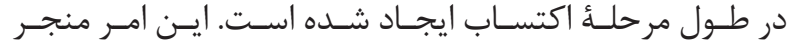

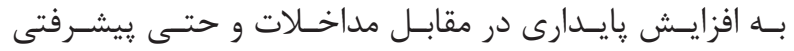

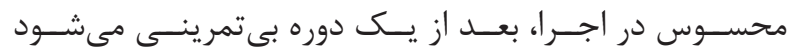

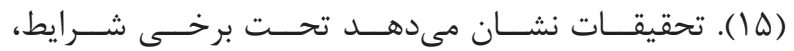

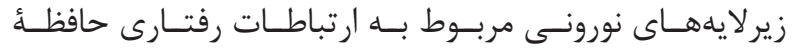

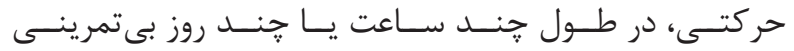

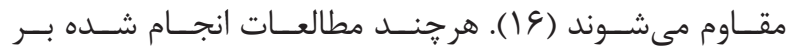

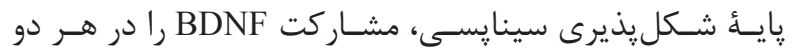

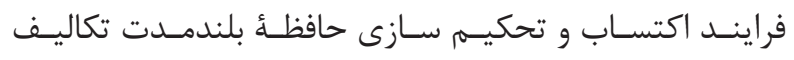

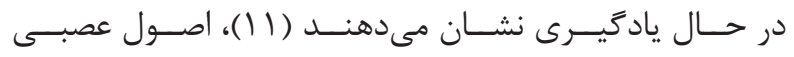

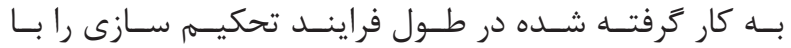

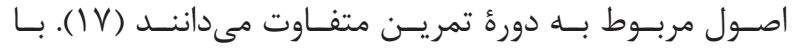

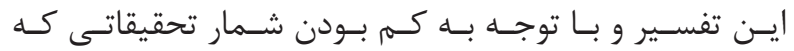

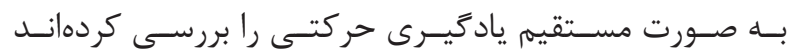

${ }^{1}$ Brain derived neurotrophic factor

${ }^{2}$ Synaptic plasticity

${ }^{3}$ Long-term potentiation

${ }^{4}$ Limbic structures

${ }^{5}$ Asingle nucleotide polymorphism

${ }^{6}$ Consolidation

${ }^{7}$ McHughen

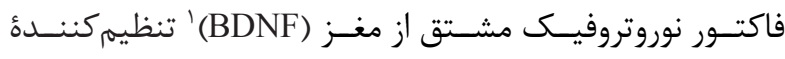

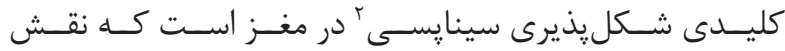

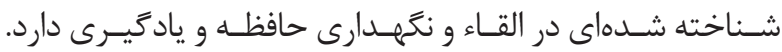

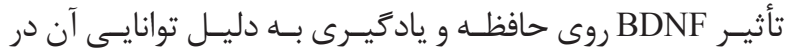

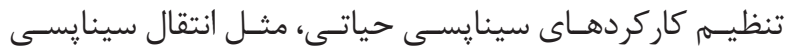

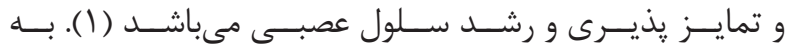

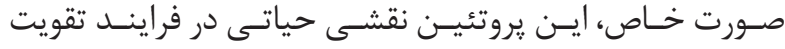

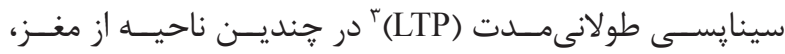

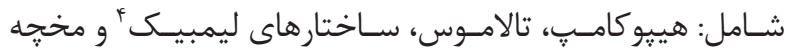

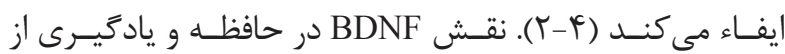

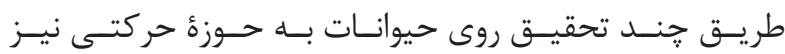

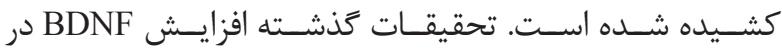

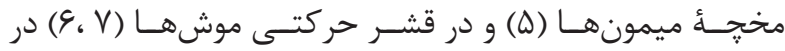

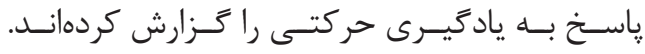

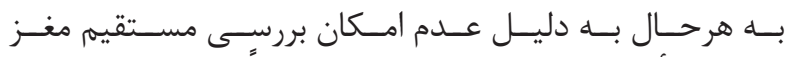

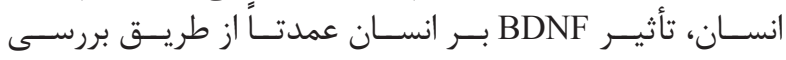

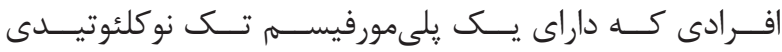

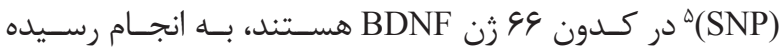

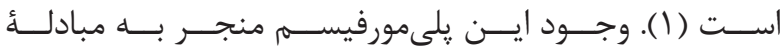

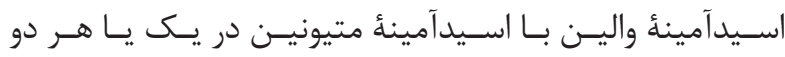

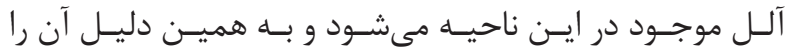

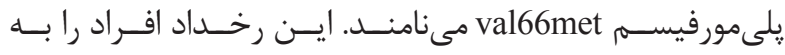

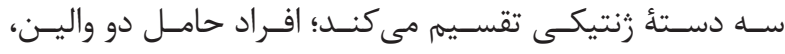

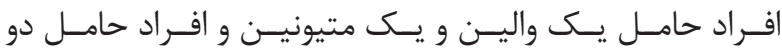

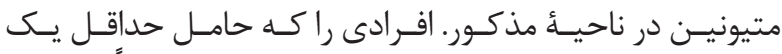

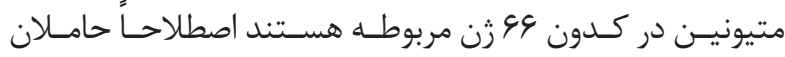

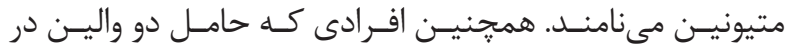

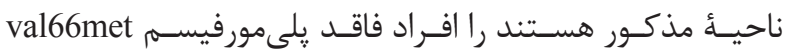

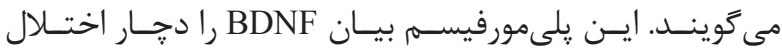

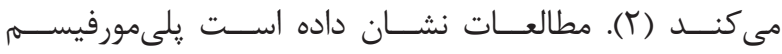

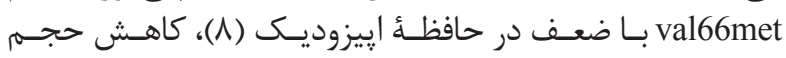

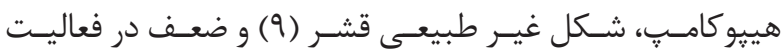

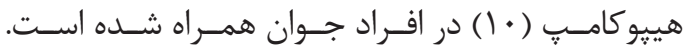

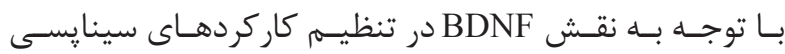

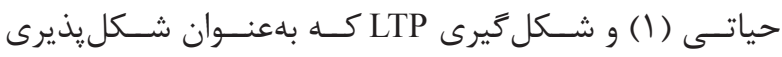

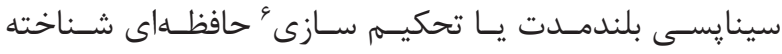

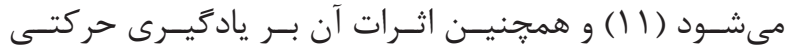

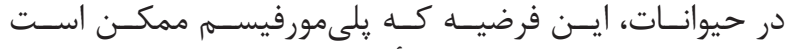

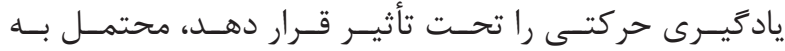

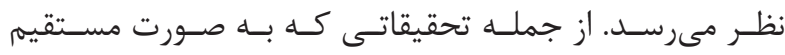

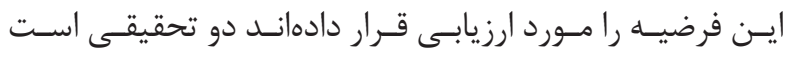

\footnotetext{
${ }^{8}$ Functional magnetic resonance imaging

${ }^{9}$ Marble navigation task

${ }^{10}$ Visuomotor adaptation task

${ }^{11}$ Blood-oxygen-level dependent

${ }^{12}$ Freundlieb

${ }^{13}$ Beste

${ }^{14}$ Response inhibition
} 


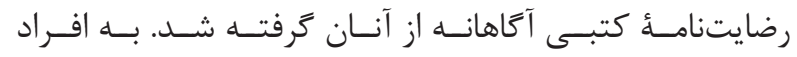

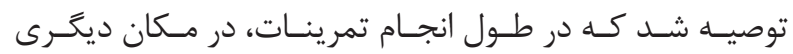

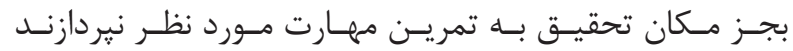

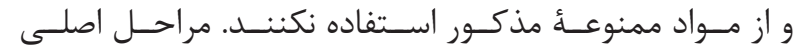

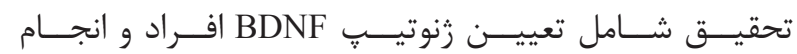

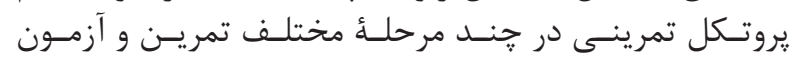

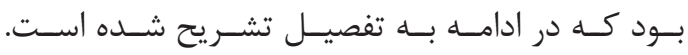

BDNF تعيين زُنوتيب

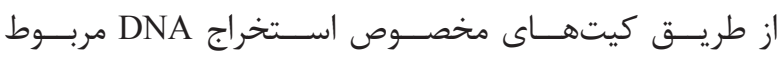

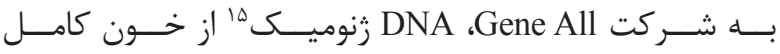

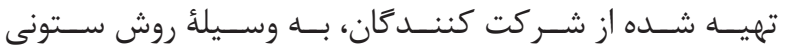

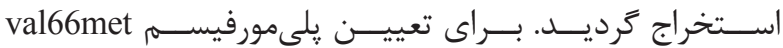

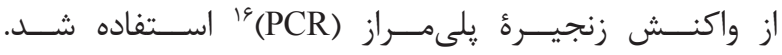

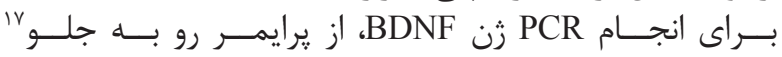
(5-ACTCTGGAGAGCGTGAAT-3) (5-ATACTGTCACACACGCTG-3)

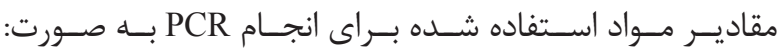

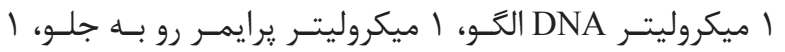

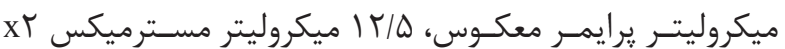

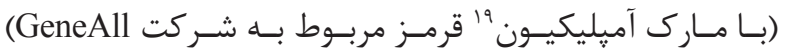

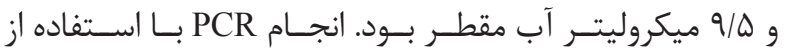

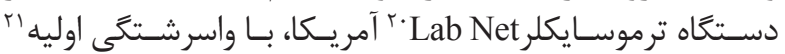

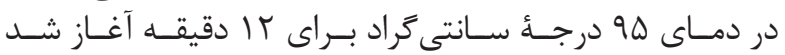

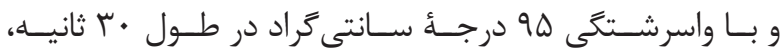

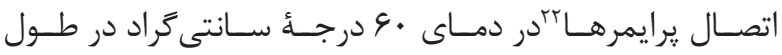

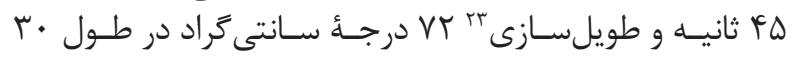

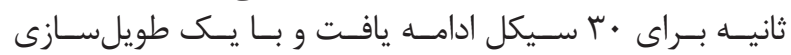

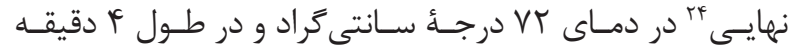

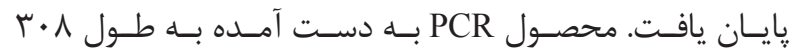

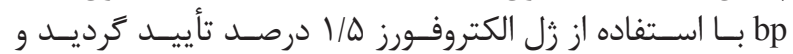

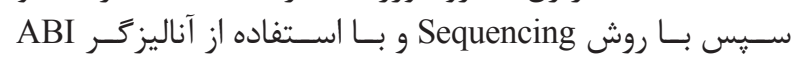
PRISM 7000 Sequencing

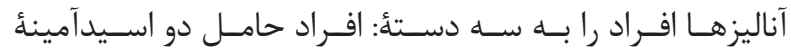

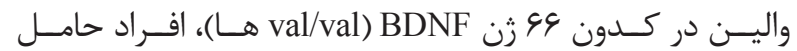

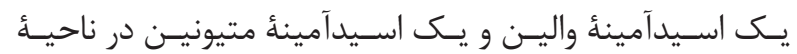

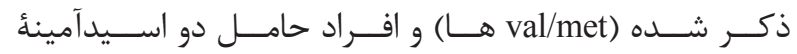

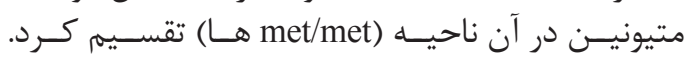

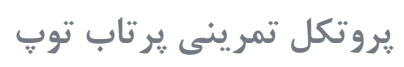

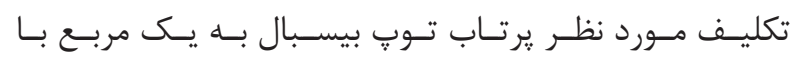

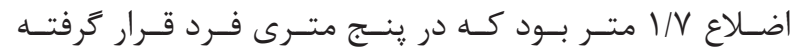

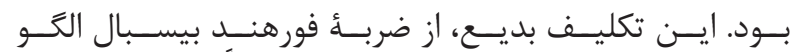

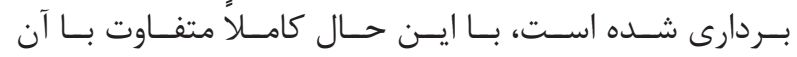

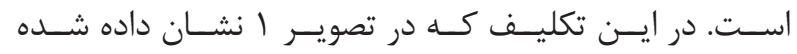

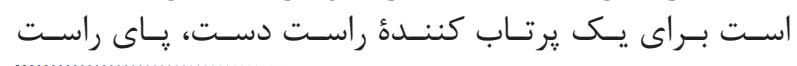

\section{${ }^{15}$ Genomic}

${ }^{16}$ Polymerase chain reaction

${ }^{17}$ Forward primer

${ }^{18}$ Reverse primer

${ }^{19}$ Ampliqun

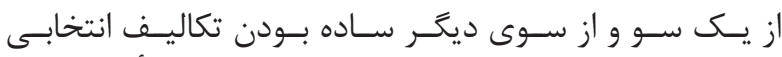

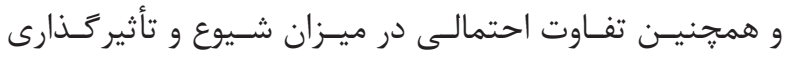

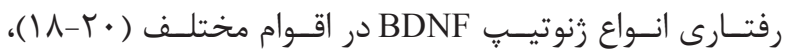

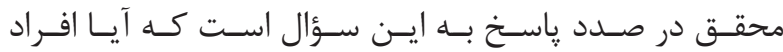

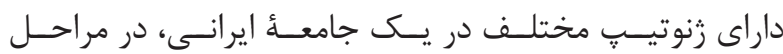

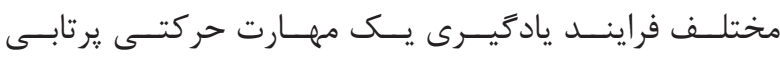

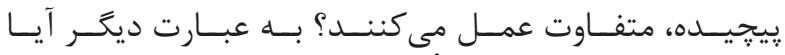

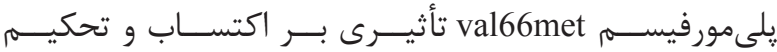

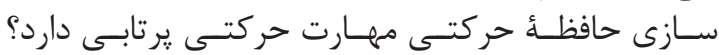

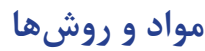
جامعه و نمونة آمارى

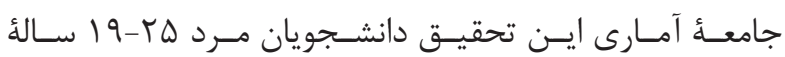

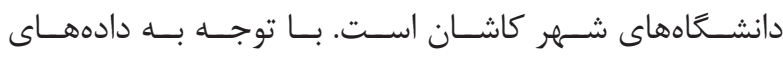

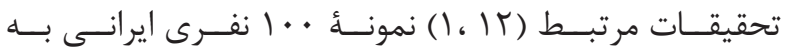

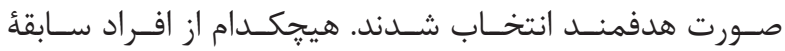

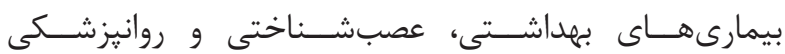

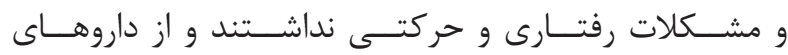

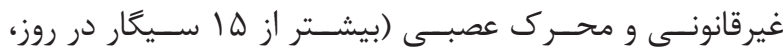

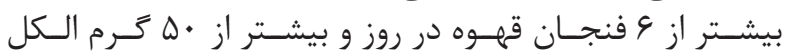

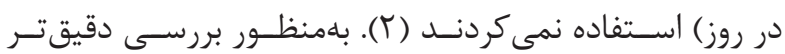

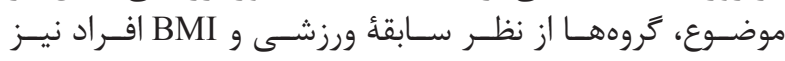

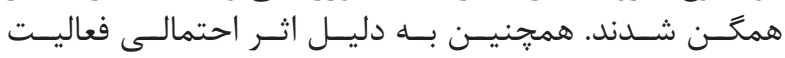

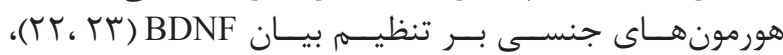

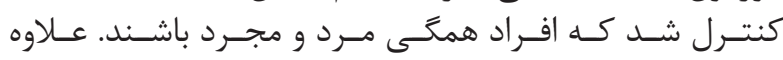

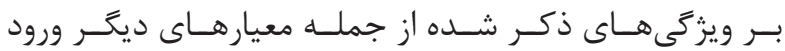

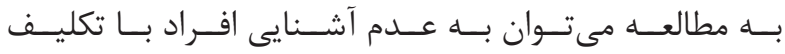

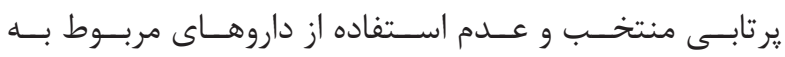

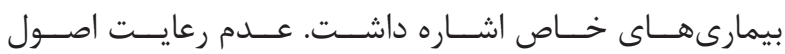

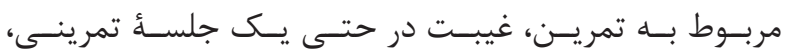

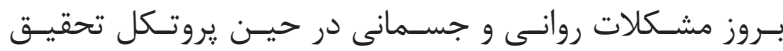

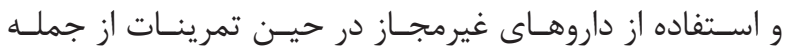

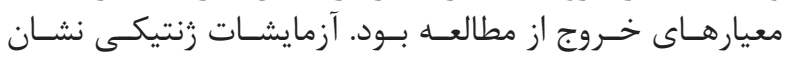

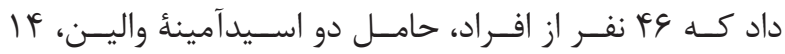

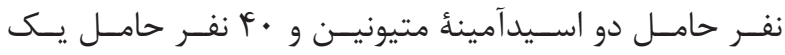

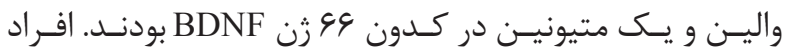

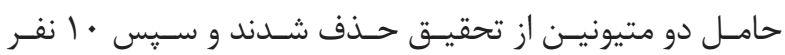

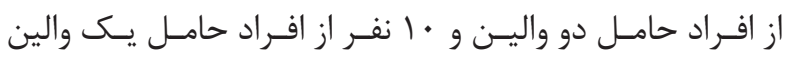

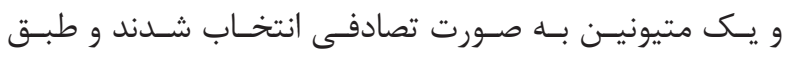

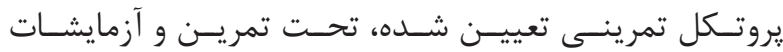

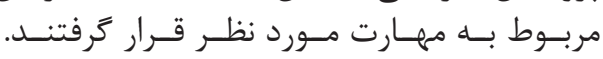

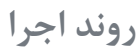

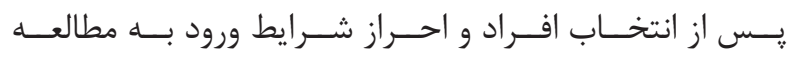

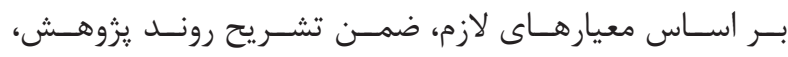

${ }^{20}$ Thermal cycler

${ }^{21}$ Initial denaturation

${ }^{22}$ Annealing

${ }^{23}$ Extension

${ }^{24}$ Final extension 


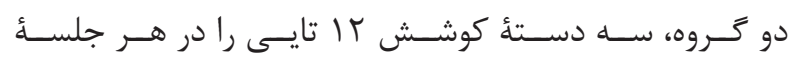

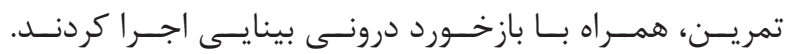

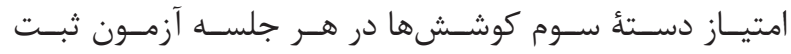

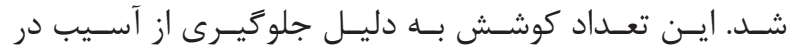

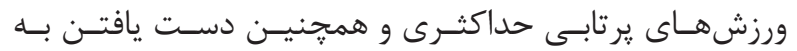

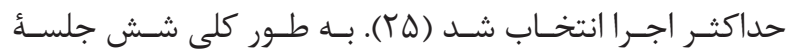

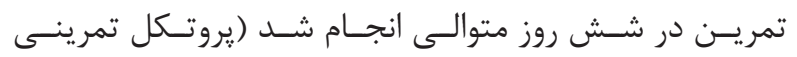

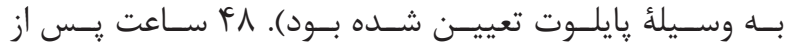

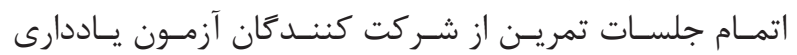

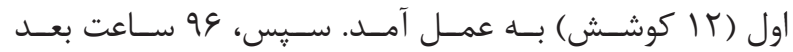

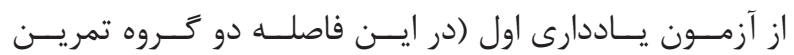

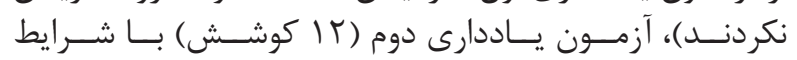

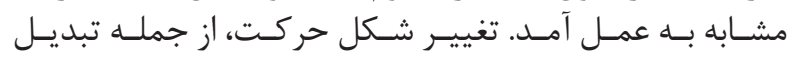

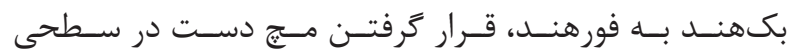

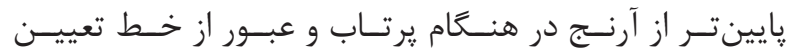

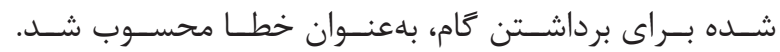

$$
\text { روش هاى آمارى }
$$

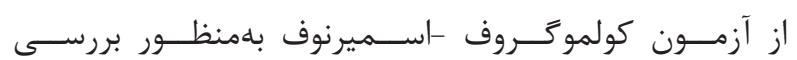

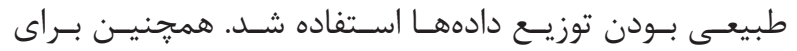

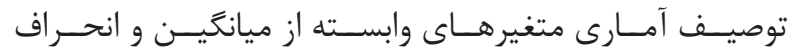

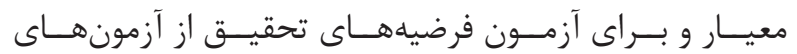

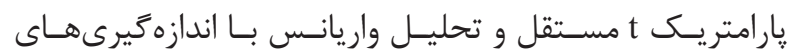

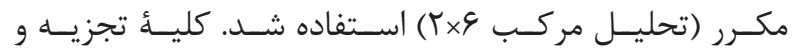

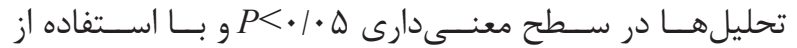

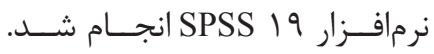

بافته ها

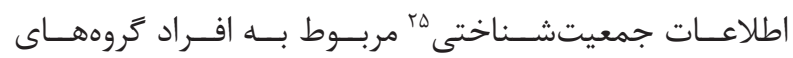

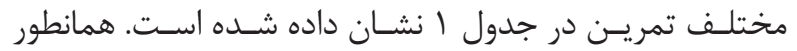

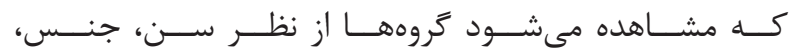

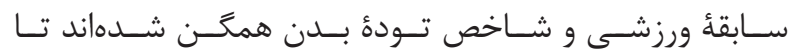

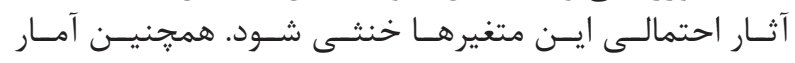

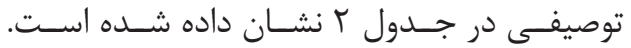

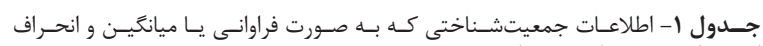

\begin{tabular}{|c|c|c|c|}
\hline حاملان هتيوئين & افراد فأقد بلمىمورغيسم & & \\
\hline 1. & 1. & \multicolumn{2}{|l|}{ تعداد } \\
\hline$r / r \cdot \pm r / \Delta$. & $\pi r r \cdot \pm \pi r$. & \multicolumn{2}{|l|}{ سن (سال) س } \\
\hline.$\wedge$. & $\cdot /$. & \multicolumn{2}{|l|}{ جنس (مرد/زان) } \\
\hline $1 \mathrm{~N} \wedge \pm \cdots \cdot \Delta$ & $1,1 y \pm \cdot . \cdot 9$ & \multicolumn{2}{|l|}{ قد (متر) } \\
\hline $9 q / / \cdot \pm 9 / 9 q$ & $V \Psi N \cdot \pm \mid r / / S$ & \multicolumn{2}{|l|}{ وزن (كيلوَّرم) } \\
\hline Vلنر & ن نقر & مدرسه يا شهرستان & \\
\hline انفر & انفر & استان & 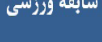 \\
\hline بانفر & بانفر & 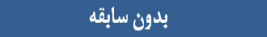 & \\
\hline r نفر & r نفر & كويكتر از +Y (كيلوَّرم بر متر مريع) & \\
\hline بنفر & ونفر & بين & BVII \\
\hline 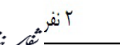 & بآنقر & 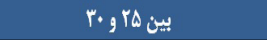 & \\
\hline
\end{tabular}

${ }^{25}$ Demographic

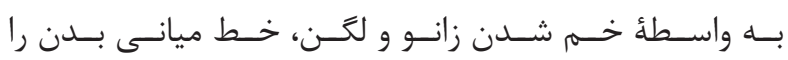

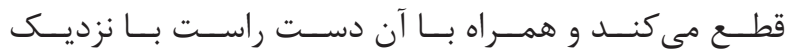

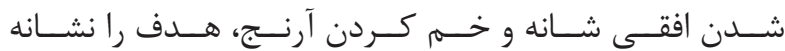

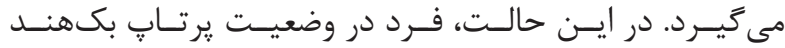

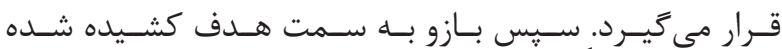

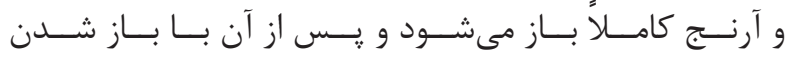

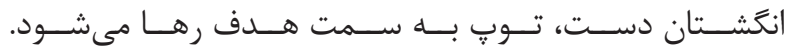

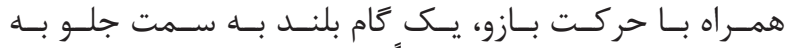

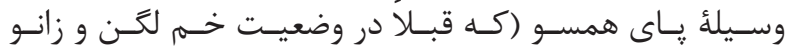

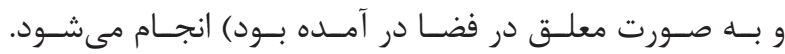

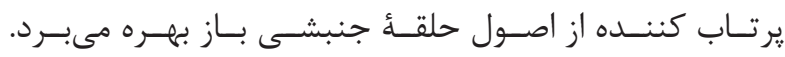

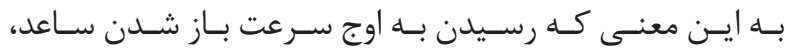

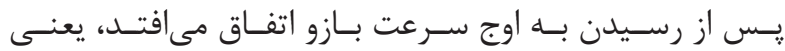

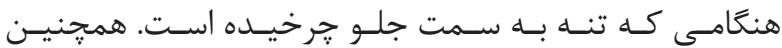

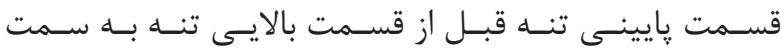

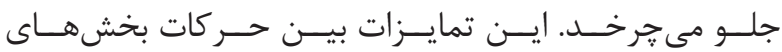

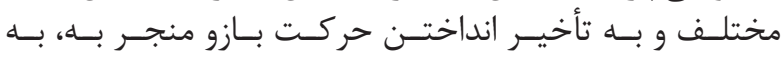

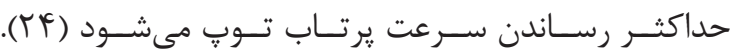

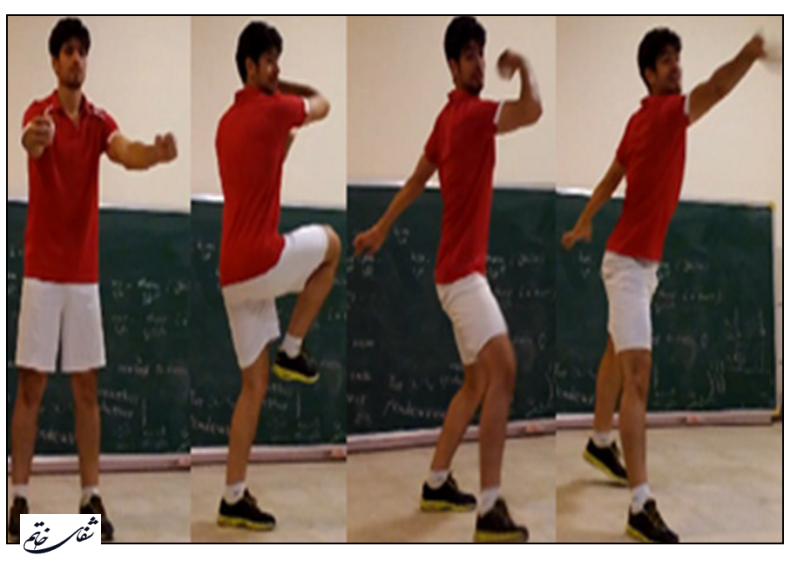

تصوير ا- نحوهُ پر تاب توض به سمت هدف كه در فاصلة له مترى فرد و روى ديوار قرار كرفته است.

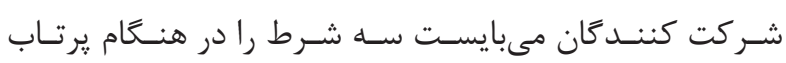

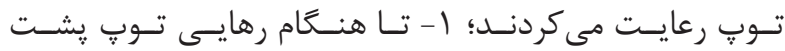

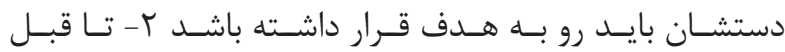

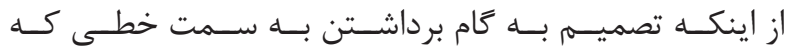

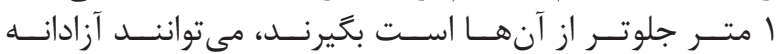

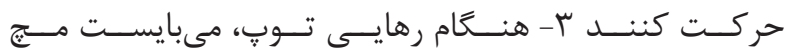

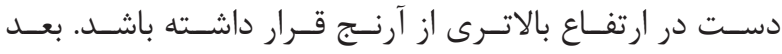

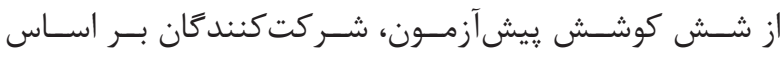

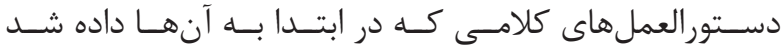

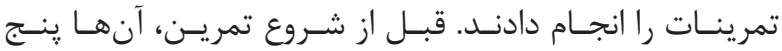

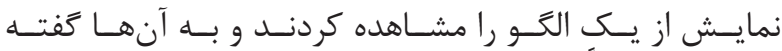

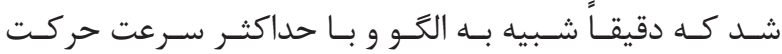

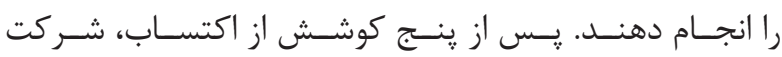

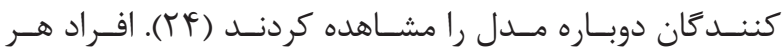


جدول r- نمرة خطاى كروهها در جلسات و آزمونهاى مختلف كه به صورت ميانكَين و انحراف استاندارد نشان داده شده است.

\begin{tabular}{|c|c|c|}
\hline حاملان متيونين & افراد فاقد يلىمورفيسم & \\
\hline$\Delta \cdot / 9 V \pm 1 \Delta / 1 T$ & $F V / r r \pm q / f V$ & نمرة خطاى يیشآزمون (سانتىمتر) \\
\hline$r V / Q \cdot \pm f / f q$ & $r q / r) \pm F / \Delta \Delta$ & نمرة خطاى جلسأ اول اكتساب \\
\hline $19 / r \Delta \pm r / \cdot r$ & $r \mid / 91 \pm r / 90$ & نمرة خطاى جلسأ دوم اكتساب \\
\hline$r \cdot / \cdot \Lambda \pm r / 9 V$ & $19 / 0 \cdot \pm \wedge / 0$ & نمرة خطاى جلسهُ سوم اكتساب \\
\hline$r \cdot / r \cdot \pm r / V G$ & $1 G / F F \pm F / K \Delta$ & نمرة خطاى جلسهُ جهارم اكتساب \\
\hline 19/9r $\pm \Delta / \cdot 9$ & $|\& / r| \pm F / r \wedge$ & نمرءٔ خطاى جلسهُ ِينجمم اكتساب \\
\hline $19 / f \wedge \pm f / 10$ & DQD/ArEF/Kr & نمرة خطاى جلسهُ ششمى اكتساب \\
\hline $19 / r f \pm r / 99$ & $\mid \& / \Delta F \pm \Delta / \Delta$. & نمره خطاى آزمون ياددارى اول \\
\hline$r r / Q V \pm f / \Delta V$ & $1 \Delta / \& \& \pm \Delta / r q$ & نمرة خطاى آزمون ياددارى دوم \\
\hline
\end{tabular}

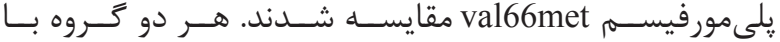

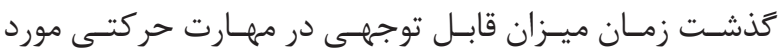

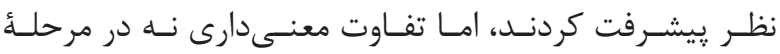

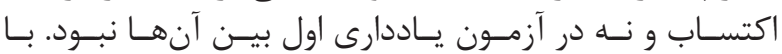

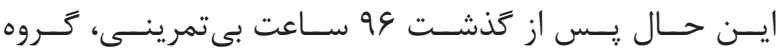

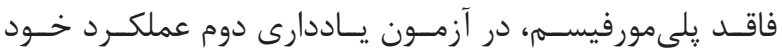

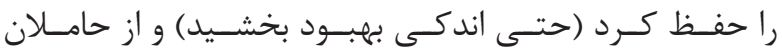

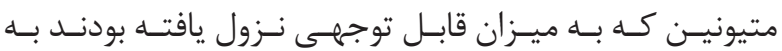

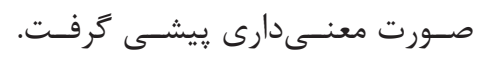

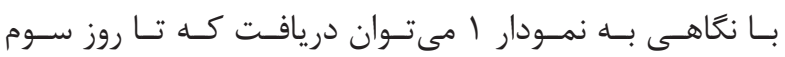

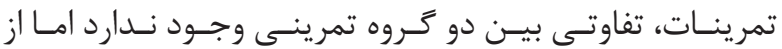

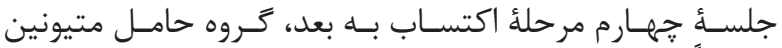

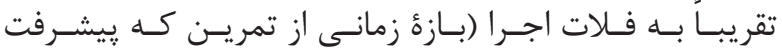

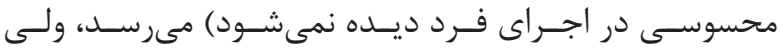

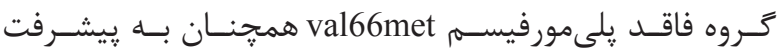

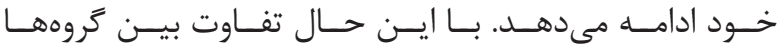

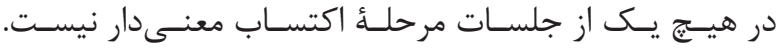

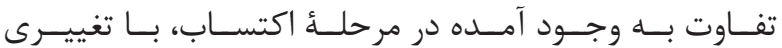

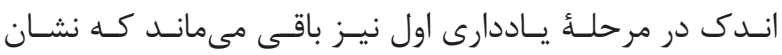

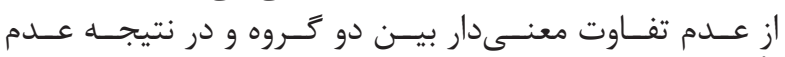

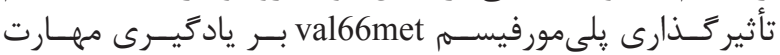

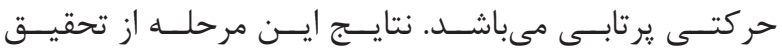

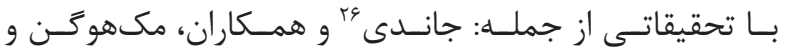

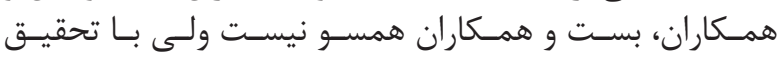

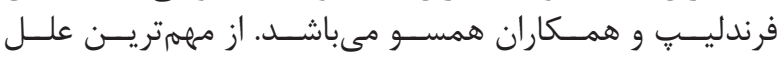

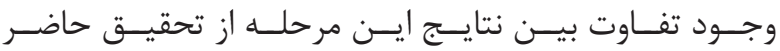

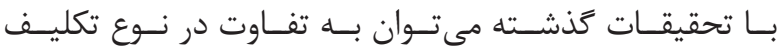

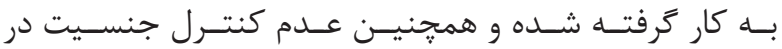

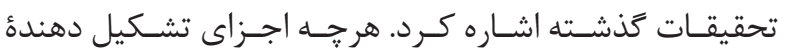

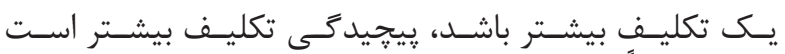

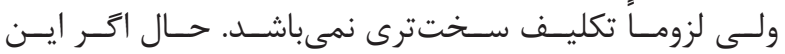

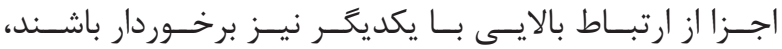

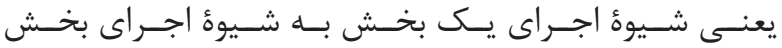

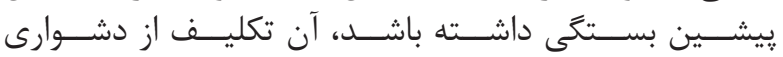

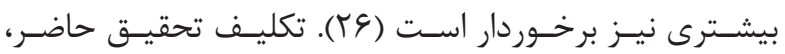
${ }^{26}$ Joundi
ييش آزمـــون: آزمــون t مسـتقل نشـان دهنــده عـدم تفــاوت

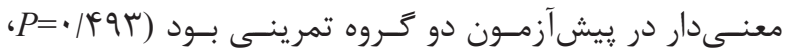
. $(\mathrm{df}=) 11)$ ، $\mathrm{t}=$. 1999

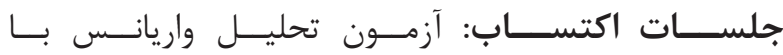

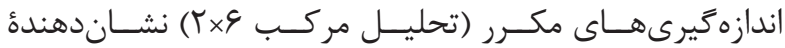

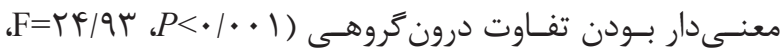

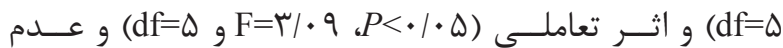

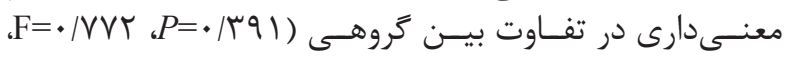
(df=1

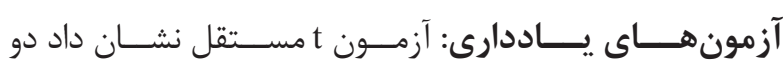

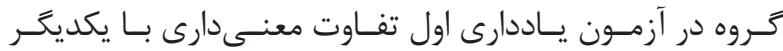

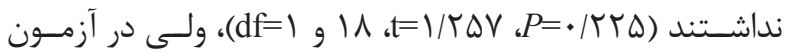

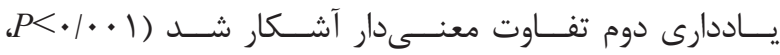

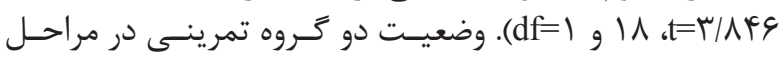

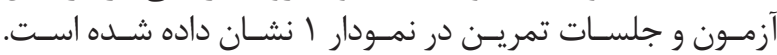

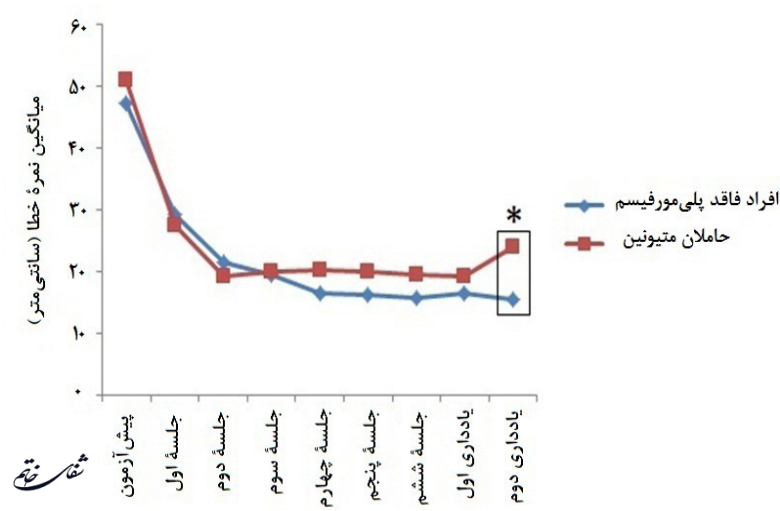

نمودار ا-وضعيت دو كروه در مراحل مختلف تمرين و آزمون. تفاوت معنى دار بين دو كروه.

بحث و نتيجه

هـدف مطالعــه حاضـر بررسـى تأثيـر ريلى مورفيسـم val66met

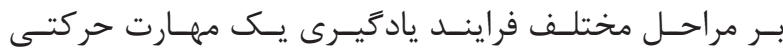

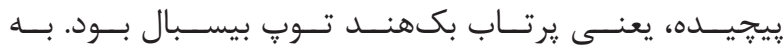

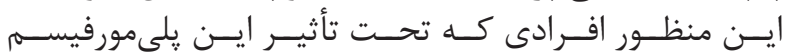

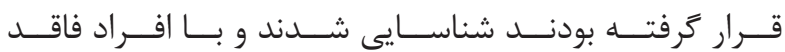




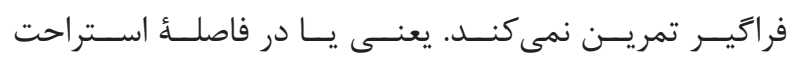

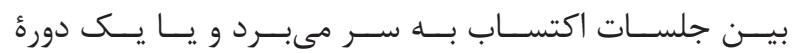

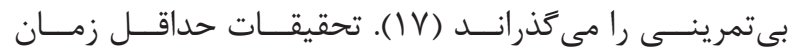

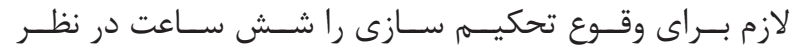

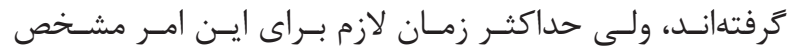

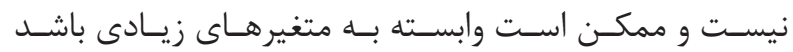

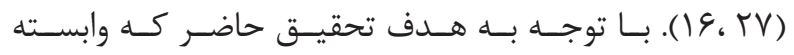

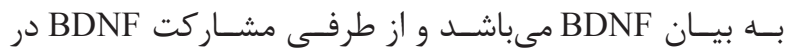

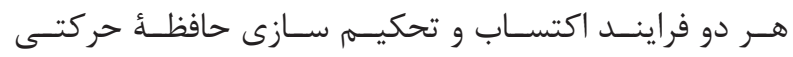

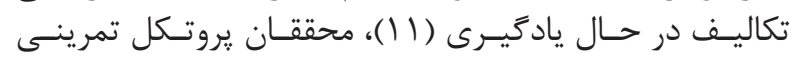

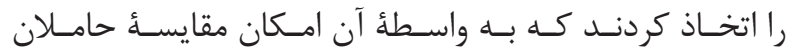

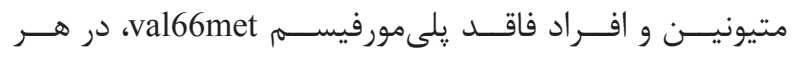

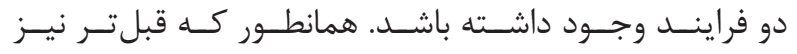

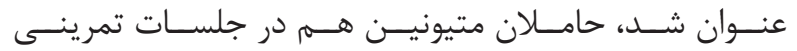

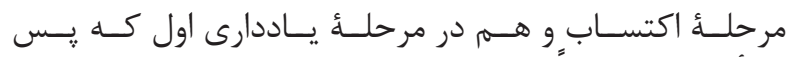

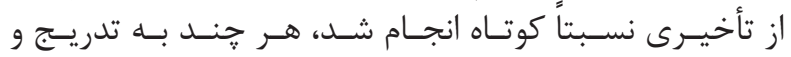

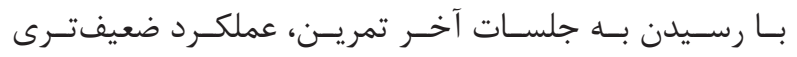

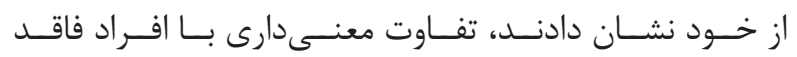

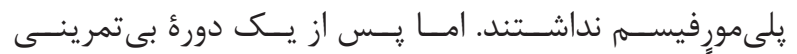

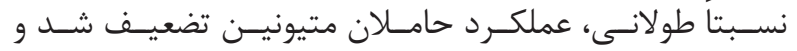

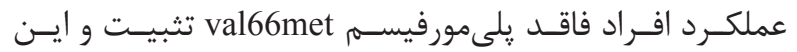

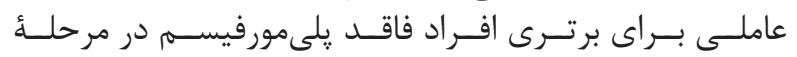

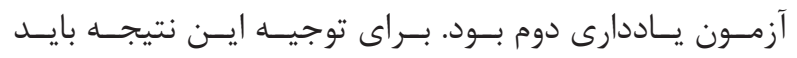

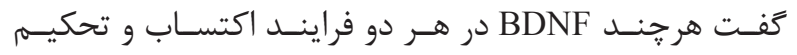

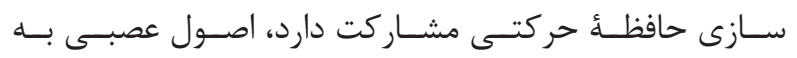

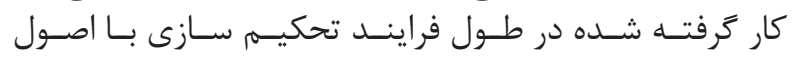

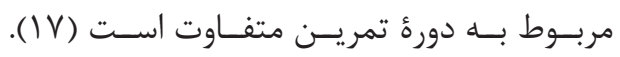

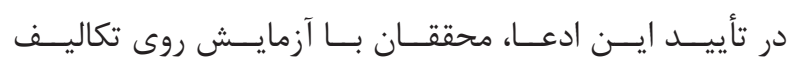

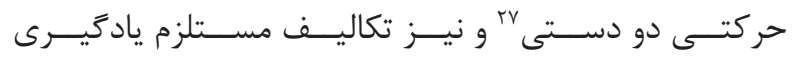

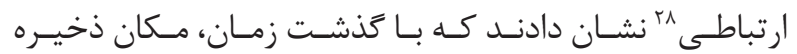

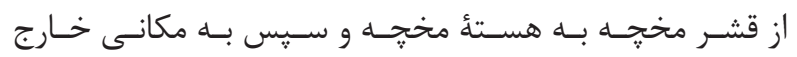

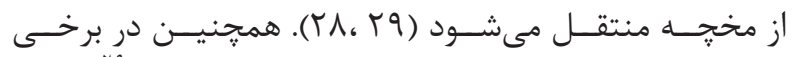

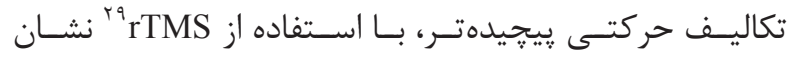

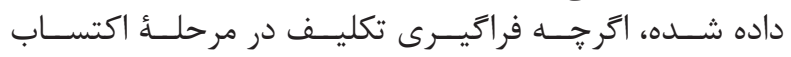

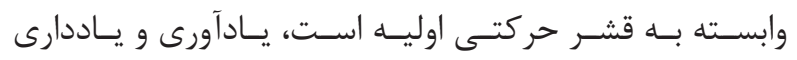

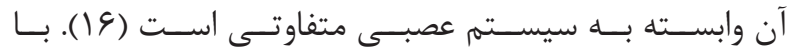

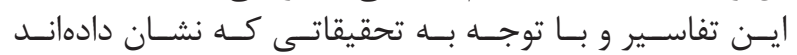

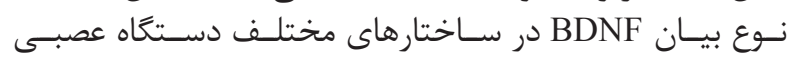

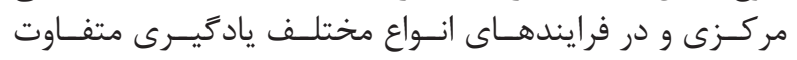

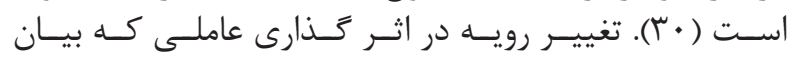

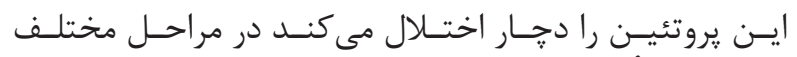

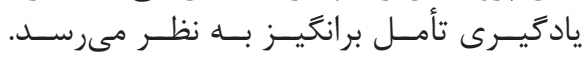

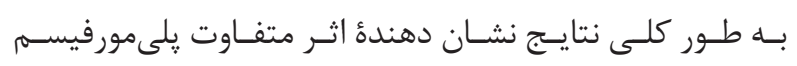

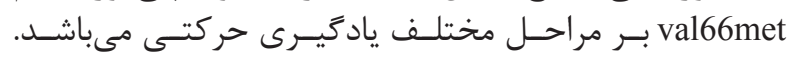

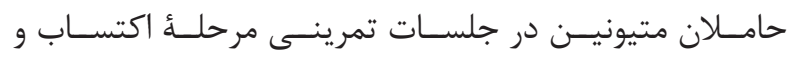

${ }^{27}$ Bimanual movements

${ }^{28}$ Associativelearning

${ }^{29}$ Repeated trans cranial magnetic stimulus

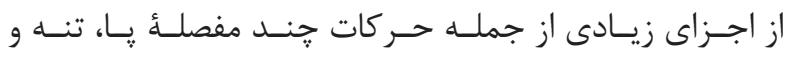

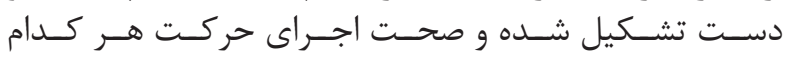

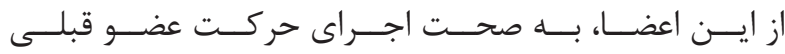

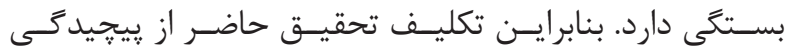

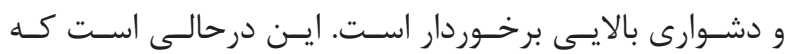

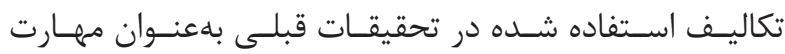

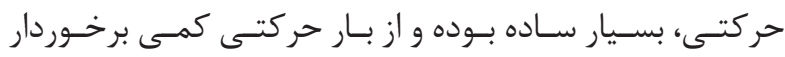

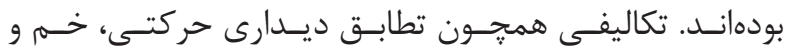

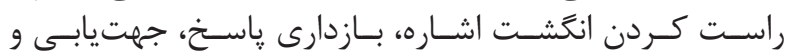

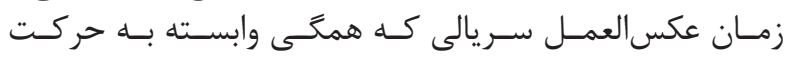

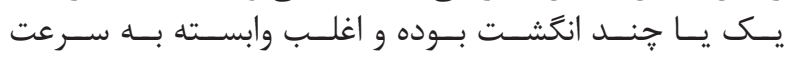

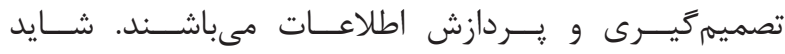

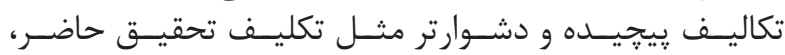

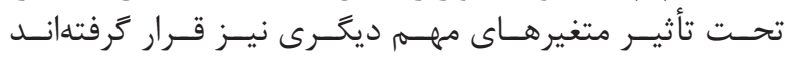

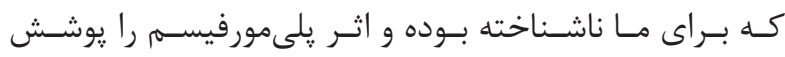

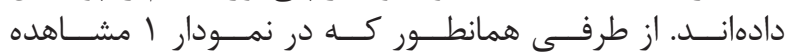

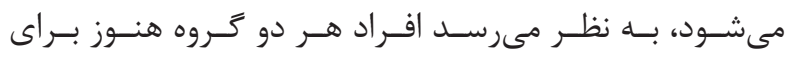

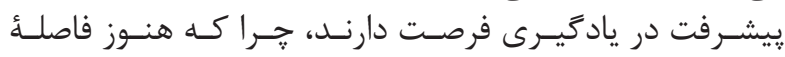

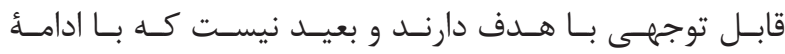

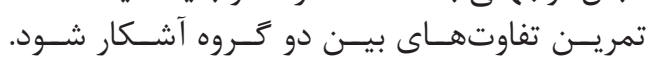

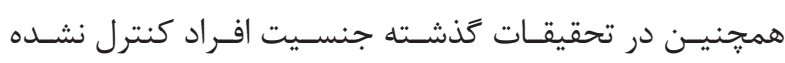

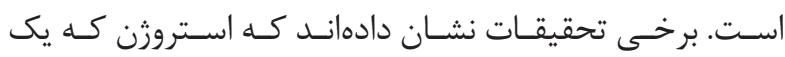

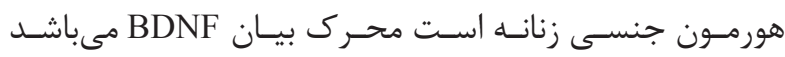

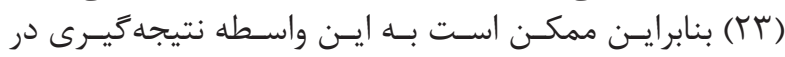

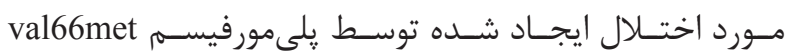

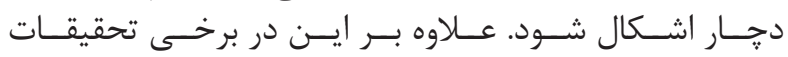

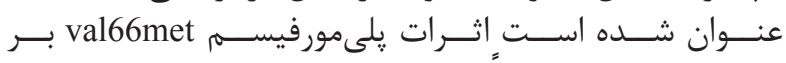

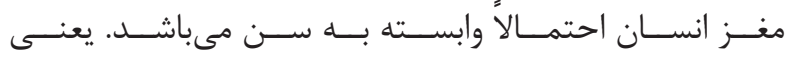

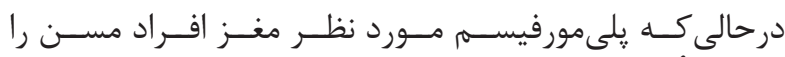

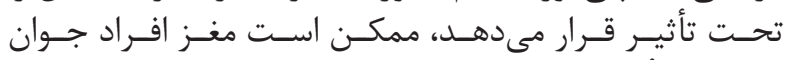

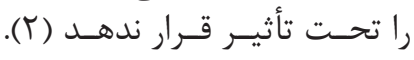

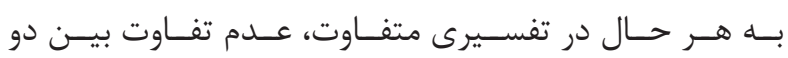

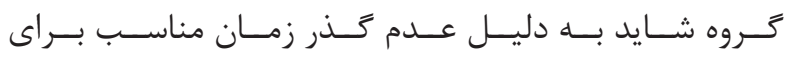

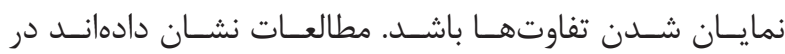

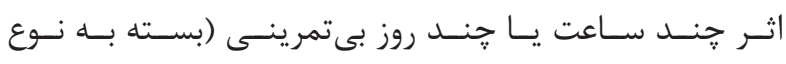

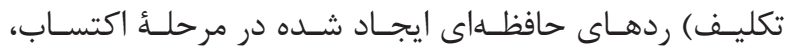

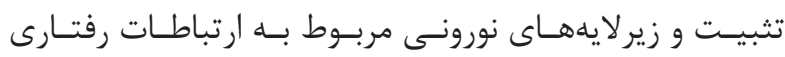

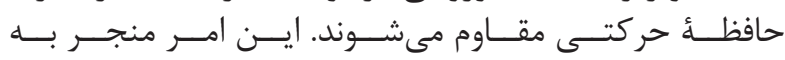

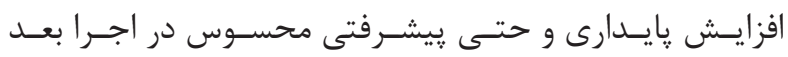

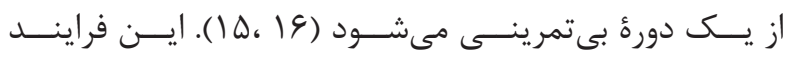

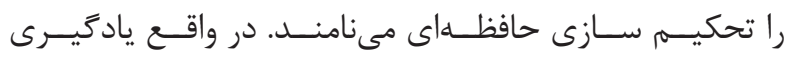

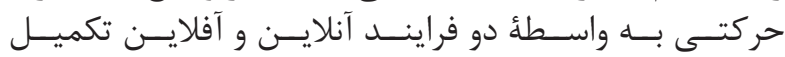

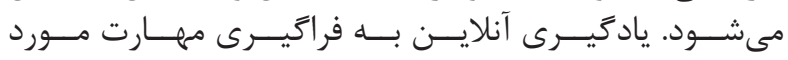

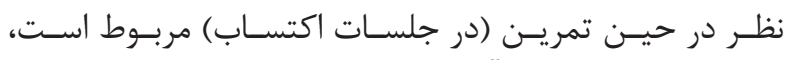

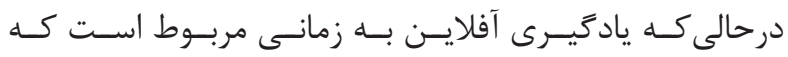




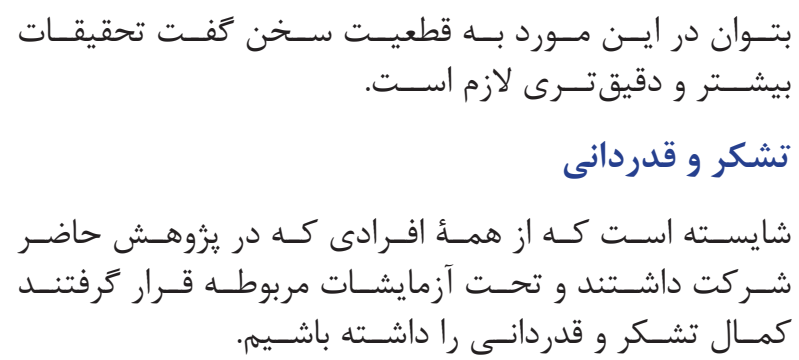

1. Joundi RA, Lopez-Alonso V, Lago A, Brittain JS, Fernandez-Del-Olmo M, Gomez-Garre P, et al. The effect of BDNF val66met polymorphism on visuomotor adaptation. Exp Brain Res. 2012; 223(1): 43-50.

2. Freundlieb N, Philipp S, Schneider SA, Bruggemann N, Klein C, Gerloff C, et al. No association of the BDNF val66met polymorphism with implicit associative vocabulary and motor learning. PLoS One. 2012; 7(11): e48327. doi: 10.1371/journal.pone.0048327.

3. Meis S, Endres T, Lessmann V. Postsynaptic BDNF signalling regulates long-term potentiation at thalamoamygdala afferents. J Physiol. 2012; 590(1): 193-208.

4. Huang ZJ, Kirkwood A, Pizzorusso T, Porciatti V, Morales B, Bear MF, et al. BDNF regulates the maturation of inhibition and the critical period of plasticity in mouse visual cortex. Cell. 1999; 98(6): 739-55.

5. Ishibashi H, Hihara S, Takahashi M, Heike T, Yokota T, Iriki A. Tool-use learning induces BDNF expression in a selective portion of monkey anterior parietal cortex. Brain Res Mol Brain Res. 2002; 102(1-2): 110-2.

6. Klintsova AY, Dickson E, Yoshida R, Greenough WT. Altered expression of BDNF and its high-affinity receptor TrkB in response to complex motor learning and moderate exercise. Brain Res. 2004; 1028(1): 92-104.

7. Bussche MVD. The role of brain derived neurotrophic factor in cortical motor learning. San Diego: University of California; 2007.

8. Egan MF, Kojima M, Callicott JH, Goldberg TE, Kolachana BS, Bertolino A, et al. The BDNF val66met polymorphism affects activity-dependent secretion of BDNF and human memory and hippocampal function. Cell. 2003; 112(2): 257-69.

9. Pezawas L, Verchinski BA, Mattay VS, Callicott JH, Kolachana BS, Straub RE, et al. The brain-derived neurotrophic factor val66met polymorphism and variation in human cortical morphology. J Neurosci. 2004; 24(45): 10099-102.

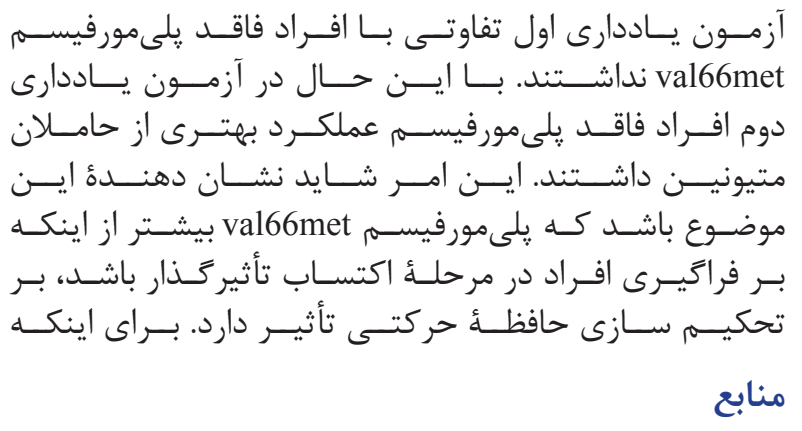

10. Hariri AR, Goldberg TE, Mattay VS, Kolachana BS, Callicott JH, Egan MF, et al. Brain-derived neurotrophic factor val66met polymorphism affects human memoryrelated hippocampal activity and predicts memory performance. J Neurosci. 2003; 23(17): 6690-4.

11. Bramham CR, Messaoudi E. BDNF function in adult synaptic plasticity: the synaptic consolidation hypothesis. Prog Neurobiol. 2005; 76(2): 99-125.

12. McHughen SA, Rodriguez PF, Kleim JA, Kleim ED, Marchal Crespo L, Procaccio V, et al. BDNF val66met polymorphism influences motor system function in the human brain. Cereb Cortex. 2010; 20(5): 1254-62.

13. McHughen SA, Pearson-Fuhrhop K, Ngo VK, Cramer SC. Intense training overcomes effects of the Val66Met BDNF polymorphism on short-term plasticity. Exp Brain Res. 2011; 213(4): 415-22.

14. Beste C, Baune BT, Domschke K, Falkenstein M, Konrad C. Paradoxical association of the brain-derivedneurotrophic-factor val66met genotype with response inhibition. Neuroscience. 2010; 166(1): 178-84.

15. Janacsek K, Nemeth D. Predicting the future: from implicit learning to consolidation. Int J Psychophysiol. 2012; 83(2): 213-21.

16. Krakauer JW, Shadmehr R. Consolidation of motor memory. Trends Neurosci. 2006; 29(1): 58-64.

17. Cohen DA, Pascual-Leone A, Press DZ, Robertson EM. Off-line learning of motor skill memory: a double dissociation of goal and movement. Proc Natl Acad Sci U S A. 2005; 102(50): 18237-41.

18. Shimizu E, Hashimoto K, Iyo M. Ethnic difference of the BDNF 196G/A (val66met) polymorphism frequencies: the possibility to explain ethnic mental traits. Am J Med Genet B Neuropsychiatr Genet. 2004; 126B(1): 122-3.

19. Bath KG, Lee FS. Variant BDNF (Val66Met) impact on brain structure and function. Cogn Affect Behav 
Neurosci. 2006; 6(1): 79-85.

20. Pivac N, Kim B, Nedic G, Joo YH, KozaricKovacic D, Hong JP, et al. Ethnic differences in brainderived neurotrophic factor Val66Met polymorphism in Croatian and Korean Healthy Participants. Croat. 2009; 50(1): 43-8.

21. Tonacci A, Borghini A, Mercuri A, Pioggia G, Andreassi MG. Brain-derived neurotrophic factor (Val66Met) polymorphism and olfactory ability in young adults. J Biomed Sci. 2013; 20: 57. doi: 10.1186/14230127-20-57.

22. Carbone DL, Handa RJ. Sex and stress hormone influences on the expression and activity of brainderived neurotrophic factor. Neuroscience. 2013; 239: 295-303.

23. Zhou J, Zhang H, Cohen RS, Pandey SC. Effects of estrogen treatment on expression of brain-derived neurotrophic factor and cAMP response elementbinding protein expression and phosphorylation in rat amygdaloid and hippocampal structures. Neuroendocrinology. 2005; 81(5): 294-310.

24. Horn RR, Williams AM, Hayes SJ, Hodges NJ, Scott MA. Demonstration as a rate enhancer to changes in coordination during early skill acquisition. J Sports Sci.
2007; 25(5): 599-614.

25. Washington RL, Bernhardt DT, Gomez J, Johnson MD, Martin TJ, Rowland TW, et al. American academy of pediatrics: risk of injury from baseball and softball in children. Pediatrics. 2001; 107(4): 782-4.

26. Magill RA. Motor learning and control: concepts and applications. 8th ed. London: McGraw-Hill Education; 2007.

27. Song S. Consciousness and the consolidation of motor learning. Behav Brain Res. 2009; 196(2): 180-6.

28. Bracha V, Zhao L, Irwin KB, Bloedel JR. The human cerebellum and associative learning: dissociation between the acquisition, retention and extinction of conditioned eyeblinks. Brain Res. 2000; 860(1-2): 87-94.

29. Diedrichsen J, Verstynen T, Lehman SL, Ivry RB. Cerebellar involvement in anticipating the consequences of self-produced actions during bimanual movements. J Neurophysiol. 2005; 93(2): 801-12.

30. Monfils MH, Cowansage KK, LeDoux JE. Brainderived neurotrophic factor: linking fear learning to memory consolidation. Mol Pharmacol. 2007; 72(2): 235-7. 